\title{
The promise of biomarkers in colorectal cancer detection
}

\author{
Asad Umar ${ }^{\mathrm{a}}$ and Sudhir Srivastava ${ }^{\mathrm{b}}$ \\ ${ }^{a}$ Gastrointestinal and Other Cancers Research Group, Division of Cancer Prevention, National Cancer Institute, \\ 6130 Executive Blvd-EPN 2143, Rockville, MD 20852, USA \\ ${ }^{\mathrm{b}}$ Cancer Biomarkers Research Group, Division of Cancer Prevention, National Cancer Institute, 6130 Executive \\ Blvd-EPN, Rockville, MD 20852, USA
}

\section{Introduction}

Reducing the burden from death and suffering due to cancer requires improving prevention and treatment efforts and incorporating early detection and risk assessment strategies into clinical practice. Early detection and screening of cancer predates a formal cancer prevention intervention studies. For example, social scientists have been involved in research determining the epidemiologic basis of and the cost-effectiveness of screening for symptomatic and asymptomatic disease. Notwithstanding the advent of newer high-throughput technologies the availability of well accepted screening tests has long been standing barriers to early cancer detection research. The early detection of cancer and risk assessment promises to increase the number of individuals whose cancer is diagnosed in a localized, curable stage, however, technological advances are constantly pushing the boundaries of early detection.

Although not fully incorporated, recent advances in high through-put areas of gene-expression microarrays, tissue arrays, protein arrays, and proteomics offer new approaches and promise a brighter future for the early detection and cancer prevention. A coordinated research effort among biomarker-development laboratories, biomarker-validation laboratories, clinical repositories, and population-screening programs have a good promise for these technologies to be implicated in clinical practice for early detection of all cancers including colorectal. Here we discuss the need for early detection of colorectal cancers (CRC) in terms of its potential im- pact on morbidity and mortality and how current technology fails to detect early lesions and how molecular markers can enhance the this detection ability.

\section{Role of biomarkers in CRC prevention}

The idea of biomarkers that can detect cancer at an early stage dates back to the discovery of markers like CEA, SV40 and major histocompatibility HLA antigen [1-3]. However, research emphasis in cancer prevention and early detection has come a long way from single tumor and single gene analysis to one of a complete molecular profiles [4-6]. These represent constantly shifting paradigms for biomarkers of early detection and risk assessment as they are at the cross roads of technological advances in molecular diagnosis and statistical and analytic tools (i.e., algorithms).

Cancer is a consequence of insult to genome from endogenous as well as environmental factors that result in genetic alterations and complex gene-environment interactions [7]. Based on these observations and others it was proposed that majority of cancers could be prevented by eliminating or modifying exposures to carcinogens [8]. Successful strategy for cancer prevention is intimately dependent upon the identification, development and validation of effective biomarkers of risk and early detection.

Overall risk for colorectal cancer varies depending on the stage of cancer and the ability to effectively diagnose at an early stage. Cancer prevention strategies 
can be as primary prevention, which is addressed to apparently healthy individuals with the goal of reducing occurrence of the disease [9]. Next level of prevention is applied to individuals affected by premalignant conditions, with the goal of reversing or inhibiting the carcinogenesis process, referred as secondary prevention. Most importantly, secondary prevention involves the identification of cohorts at greater than average risk for disease through screening for sub-clinical pathology followed by interventions to arrest progression, hence cancer biomarkers are a foremost part of this detection effort. Finally, tertiary prevention involves patients with diagnosed disease in whom applied interventions may improve, alleviate and reduce complications [10].

\subsection{Biomarkers in cancer prevention}

Each level of cancer prevention is highlighted by biomarkers of risk, early detection and exposure. Further, the field of cancer prevention has tremendously benefited from surrogate endpoint biomarkers. Each of these biomarkers and their role in early detection and risk assessment is discussed below.

In addition biomarkers for cancer prevention must also be validated before they can have an impact on cancer risk assessment. The need to characterize a set of valid biomarkers that reflect events relevant to carcinogenesis is a leading priority in cancer prevention research. Validity is a general concept that refers to a range of characteristics of the biomarker, such as its determinants or the analytic procedures. An impressive amount of literature has been published on the concept of biomarker validation and the various aspects of the validation process. For the review of biomarker literature see [11-13].

\section{Biomarkers of risk}

It is important to distinguish between markers of risk and markers of early detection as they share the same outcome, namely, the incidence of disease (cancer). Markers of risk indicate that cancer is more likely to occur in certain persons compared to general population. A positive risk marker confers significantly less than $100 \%$ certainty of cancer within a specified time interval, whereas early detection markers on the other hand confer close to $100 \%$ certainty of cancer.

All surrogate markers that are not diagnosed with cancer incidence are by definition biomarkers of risk. For example, for CRC polyps and adenomas are sur- rogate endpoint biomarkers, as polyp/adenoma incidence does not indicate cancer incidence but indicates an increased risk for colon cancer. To decide which risk factors can be used as surrogate endpoint biomarkers, the risk marker and its method of detection has to be well defined and knowledge of its strength and relationship to the definitive outcome must be known. Some putative biomarkers of risk is listed in Table 1. Biomarkers as surrogate endpoint are used primarily to save cost and time in intervention trials. These criteria for biomarkers be tested and evaluated in preclinical models using cell, tissue culture and animals.

There are several factors that have been described for an increased risk of CRC incidence. As described above biomarkers of risk can be inherited or acquired. First and foremost is the age, the risk of CRC begins to increase after the age of 40 years and rises sharply at the ages 50-55 years; the risk doubles with each succeeding decade, reaching a peak by 75 . One can argue this association for an increased risk either due to inherited infidelity of normal replication process [14-16] or due to an accumulated mutational effect of environmental risk factors. Most likely, it is an interaction of both risk factors giving sometimes a synergistic outcome in terms of altered genomes and pathways.

Many affected genes and their roles in dysfunctional pathways in CRC have been identified by analyzing sporadic CRC and FAP cancers. Recently, the role of DNA mismatch repair pathway, which plays a critical role in the maintenance of genomic integrity during replication process, has been identified as the key dysfunction in HNPCC cancers [17-19].

It has been reported that a Western lifestyle (associated with diet and physical activity) shows a positive correlation with a high risk of CRC [20]. For example, a case control study indicated that Western lifestyle was associated with chronic levels of insulin and IGF1 in the serum and high risk of CRC. It is likely that other dietary agents that reduce the risk of CRC interact with either colonic bacteria, bile acids, mucins and carcinogens at different levels affecting the overall risk of cancer incidence.

\section{Biomarkers for early detection}

Early detection biomarkers that can identify cancer using either serum or feces are ideally suited as a reliable screening tool which has an underlying goal of "early diagnosis and risk assessment" [21]. In order for a biomarker to be useful in clinics routinely, several 
economic and technical limitations need to be considered in terms of their effectiveness and predictive value. However, no such single biomarker exists but research holds promise in that a number of tumor biomarkers are being tested and being evaluated. It is likely that a panel of biomarkers; combination could give a better predictive value for early detection. This approach was successfully used in a proteomic profiling detection of the risk of ovarian cancer and fecal DNA panel in CRC [22].

Realization of colorectal carcinogenesis as a continuum of molecular aberrations rather than a single cataclysmic event has led to major shifts in the paradigms for early detection and cancer prevention. Molecular aberrations in phenotypic steps of CRC seem to accumulate with advancing histopathologic lesion (i.e., aberrant crypts to adenomas to carcinoma). At each of these steps there is an opportunity for detection and intervention - a chance to prevent, slow or even halt the gradual march - of healthy cells toward malignancy. It is estimated that the majority of cancers are induced by carcinogens; thus, if exposure to carcinogens is avoided, cancer can be prevented. A list of molecular abnormalities include: $k$-ras, APC, DCC, $\beta$-catenin, $M S H 2, M L H 1, S M A D 4$ and p53, among others (see Tables 1-4 and Fig. 1). Many of these mutations are currently being evaluated as potential biomarkers for early detection.

\subsection{Biomarkers of exposure}

Biomarkers of exposure provide with the measurement of internal dose estimates for individual differences in carcinogen uptake and metabolism, and provide an estimate of the actual amount of the agent within the body.

Indicators that can be used in cancer prevention are the biomarkers of exposure and/or events in biological systems or samples. Potentially relevant markers for cancer chemoprevention include exposure, intermediate, drug effect, tumor, and susceptibility biomarkers. Although characteristics and issues associated with each type of biomarker are different there is no distinct boundary between them and one type of biomarker can be used for another purpose (e.g., DNA damage as a marker for mutations in tumor suppressor genes).

For the purpose of cancer prevention discussion, a biomarker of exposure is any indicator that reflects endogenous or exogenous exposure to carcinogenic risk factors and it can be measured in the body or its products (body fluids etc) and it is predictive of the inci-
Table 1

Biomarkers for early detection

\begin{tabular}{l}
\hline Single markers \\
APC mutation \\
$p 53$ mutation \\
K-ras mutation \\
$\beta$-catenin mutation \\
Bcl-2 mutation \\
Cyclin D1 \\
Cyclin E1 \\
SMAD4 \\
Kinases \\
Proliferation in Aberrant Crypt Foci \\
MMR defects \\
$\quad h M S H 2$ \\
$\quad$ hMLH1 \\
hMSH6 \\
hPMS2 \\
hPMS1 \\
$\quad$ Microsatellite Instability (MSI) \\
Methylation of $h M L H 1$ promoter \\
Fecal Occult Blood Test \\
Panel of Biomarkers \\
Fecal DNA markers \\
Gene Expression profiles \\
Proteomic profiles \\
Methylation profiles \\
\hline
\end{tabular}

Table 2

Putative biomarkers for detection

\begin{tabular}{lll}
\hline Marker (Gene/Protein) & Mutation Incidence & Specimen \\
\hline APC & $\sim 70 \%$ & Stool \\
p53 & $50-70 \%$ & Stool \\
K-ras & $50 \%$ & Stool \\
CTNNB1 & $4-15 \%$ & Tissue \\
SMAD4 & $16 \%$ & Tissue \\
SMAD2 & $6 \%$ & Tissue \\
MSH2 & & Stool \\
MLH1 & & Stool \\
MSI & $15 \%$ & Stool, Serum \\
DCC & $3 \%$ & Tissue \\
Src & $2 \%$ & Tissue \\
IGF-1 & & Serum \\
CEA & & Serum \\
Ploidy & $80-85 \%$ & Serum \\
Methylation & & Stool, Serum \\
\hline
\end{tabular}

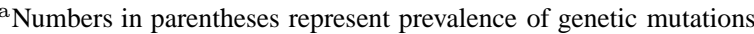
reported in [4]. b Specimens listed are those in which the assay for mutations can be performed.

dence or outcome of disease. For example, a cancer susceptibility biomarker is an indicator of a inheritable ability of carcinogenic agent or event.

Although, no exposure markers are currently used for CRC exposure risk, liver cancer serves as a model for the study of biomarker of exposure. For example, it has been well documented that exposure to aflatoxin B1 (AFB1) and hepatitis B virus (HBV) are the most important exposure risk factors for liver cancer [23,24]. Using specimens that have been collected from individ- 
Table 3

Future directions for biomarkers in cancer prevention

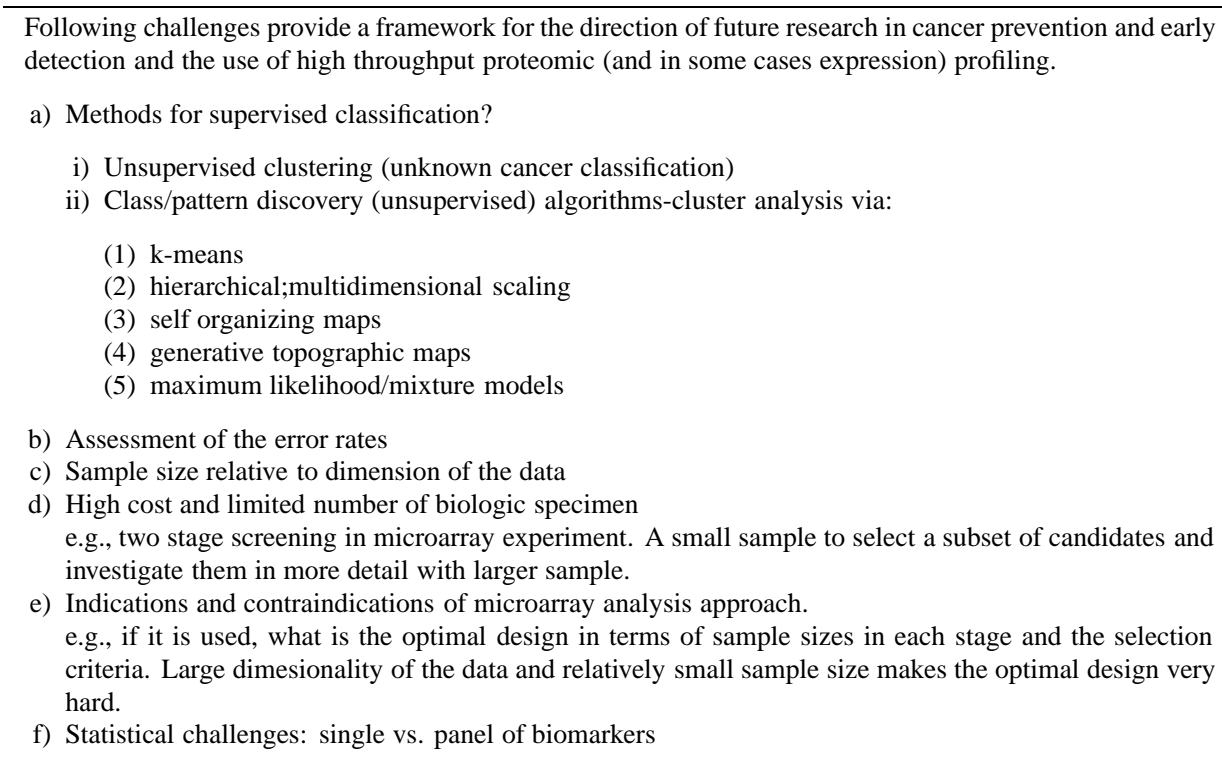

i) Are panel of biomarkers superior to single marker?

ii) Biomarkers as surrogate endpoint-theoretical advantages of shorter trial duration; stop when reached surrogate; smaller sample size, however, the challenge is to identify what data are necessary for validation, what validation statistics is best, what values of statistics indicates surrogate is acceptable?

Table 4

The criteria for the evaluation of biomarkers of risk and early detection

Criteria for Risk Biomarkers
- differentially expressed in premalignant or high-risk, and tumor tissue
- provide acceptable predictive accuracy for risk or for the presence of cancer
- the variation of the detection tests should be known
Criteria for Surrogate Endpoint Biomarkers in Prevention
- be a determinant of outcome
- modulated by chemopreventive agents
- modulation or elimination of the risk marker must correlate with a decrease in cancer incidence

uals before they developed liver cancer, the AFB1-N7guanine adduct can be found to be predictive of liver cancer. Eventually, this validated data led to the development of cancer prevention program that targeted the reduction of dietary AFB 1 and the vaccination against HBV. This exposure biomarker in hepatocellular carcinoma exemplifies the usefulness of successful cancer prevention strategy that can serve as a model for CRC cancer prevention program.

\subsection{Surrogate endpoint biomarkers}

Surrogate endpoint biomarkers are intended to substitute for a clinical endpoint, e.g., incidence of disease, death etc. The idea of surrogacy has now been extended to include "surrogate sites" - documenting changes in readily accessible anatomic sites which can serve as substitute for a clinical outcome in less accessible sites, e.g., oral-pharyngeal mucosa as a surrogate for lung cancer.

Surrogate endpoint biomarkers are an important aspect of the chemopreventive drug development process. Surrogate markers provide means for performing chemoprevention trials [25]. In the absence of surrogate endpoint biomarkers, typical clinical trials for measuring cancer incidence reduction could take 5-10 years requiring huge sample size (subject accrual in the tens of thousands) [26] with lengthy follow-up periods and high cost. Hence, the use of phenotypic and genotypic biomarkers as surrogate endpoints for cancer incidence is gaining momentum for the evaluation of chemopreventive efficacy in most cancer targets in 1-3 


\section{CRC with Chromosomal Instability (CIN)}

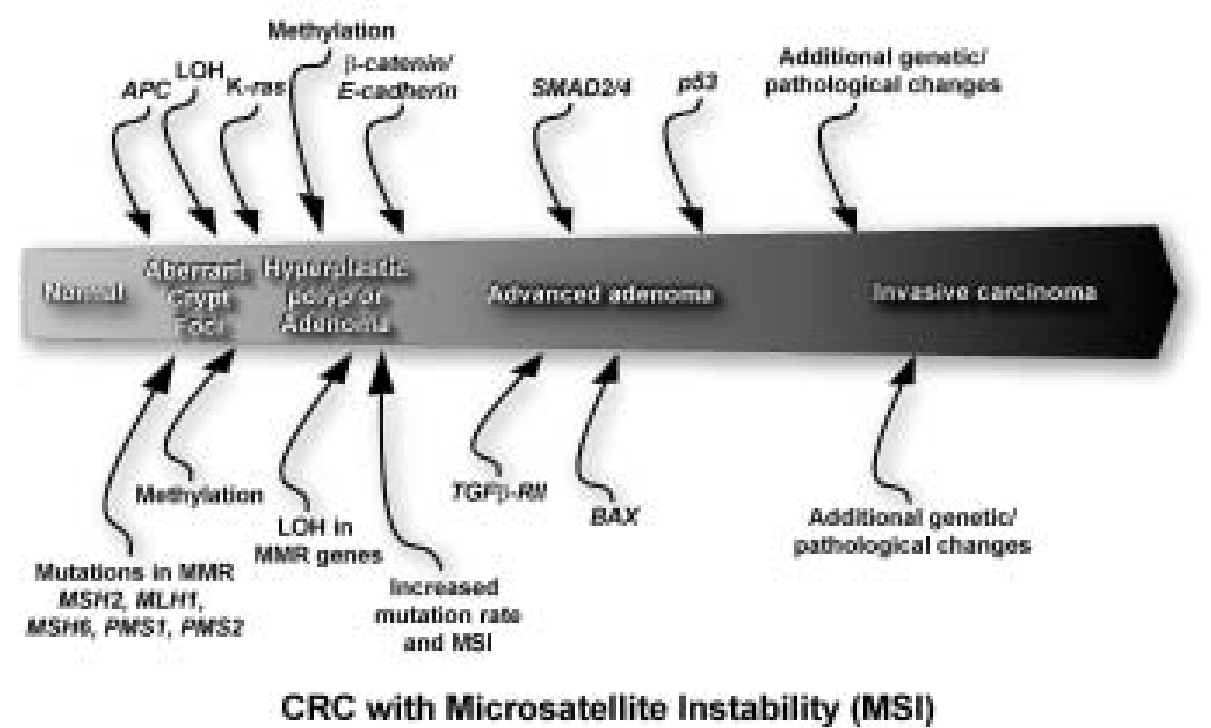

Fig. 1.

years with few hundred subjects, reducing the overall cost of chemoprevention trials.

Surrogate endpoint biomarkers are of utmost importance in preclinical and clinical prevention schemes. These biomarkers must fit expected pathological criteria of disease (i.e., altered in high risk situations), modulated by intervention strategies, readily quantified and correlate with altered disease situations (precancer/cancer) $[27,28]$.

\section{Multiple biomarkers: One for all or all for one}

Current debate for biomarkers for risk and early detection seems to be focusing on if there is a single comprehensive biomarker that can successfully predict risk for any cancer type or is there should be multiple biomarkers for a single cancer. It seems like that jury is leaning more toward the later approach as multiplexing methods are becoming more of a norm than an exception.

Recent advances in molecular biology, and specimen handling has led to the development of a panel of biomarkers to be tested simultaneously [29]. The basis of this test became apparent after it was realized that exfoliated cells from colonic mucosa are sloughed into the stool. DNA from the stool can be isolated and subjected to a multi-target DNA analysis panel. This assay can currently, assess 15 mutational hot spots including $k$-ras, p53, APC, BAT-26, and L-DNA (large DNAfragments as large as $2.4 \mathrm{~kb}$ have been observed) [29, 30].

\section{Validation of biomarkers}

Although in practice, modulable intermediate biomarkers play an important role in the evaluation of chemopreventive agents in phase II trials. However, the biggest hurdle in the advancement of biomarkers come from inability to corroborate these biomarkers in clinical setting, as these biomarkers have not been validated at any levels, especially in relation to subsequent incidence of cancer, which is the main reason that these are not being used in phase III trials.

For the validation process, questions are raised at each step and include:

1. Does the intervention strategy affect the biomarker?

2. What the percentage of affected population (cohorts) that show biomarker consistently?

3. Is the molecular mechanism as the basis for the biomarker use is warranted?

4. Is the biomarker associated with the outcome of cancer?

5. How relevant is the modulation of biomarker as an indiactor for change in disease (cancer) status? 
6. Is the biomarker associated with a chemopreventive agent or risk factor for cancer?

7. Are the risk factors also mediated by the biomarker?

The use of uncorroborated biomarkers in cancer prevention is a dangerous proposition. This can lead to not only use of spurious inferences and generalizations but can eventually lead to erroneous cancer detection and even use of failed intervention strategies. For a comprehensive review for validation of biomarker readers are referred to Srivastava et al., 2001, and Srinivas et al., $2001[11,12]$.

\section{Current knowledge of molecular alterations in cancer is the tip of the iceberg}

Although majority of colorectal cancers are sporadic in nature, however, molecular alterations that are seen in the hereditary CRC are also present in sporadic cancers. Invasive colorectal cancer is a multistep, multistage process that evolves over several years. Multistep nature of CRC also yields distinct intermediate lesions with specific molecular alterations that parallel histopathologic changes from normal epithelium, to hyperplasia, to polyp, to adenomas and carcinoma [31, 32].

Current knowledge of molecular alterations that are important for CRC include epigenetic and genetic changes in key tumor suppressors and oncogenes is extensive, however, it still represents the tip of the iceberg of knowledge that need to be resolved for a complete understanding of the CRC pathogenesis, especially the natural history. Genetic changes include alteration in overall DNA content (aneuploidy or change in DNA index), nuclear aberrations, and altered protein expression. Specific alterations were first identified systematically by Fearon \& Vogelstein and others, include $k$-ras, APC, $\beta$-catenin, MSH2, MLH1, MSH6, PMS2, DCC/SMADs, and p53 among others [31-33]. Although our understanding of CRC carcinogenesis is detailed and many steps are clearly defined, but it is far from complete. Current research in identifying molecular targets and deciphering their role in the natural history of the disease is the key to biomarker research in the future.

\section{Chromosome aberrations as cancer predictors}

It is been proposed that chromosomal aberrations are associated with cancer and can act as an inter- mediate step in the causal pathway to becoming cancer. For example, in certain cancers chromosomal rearrangements play an important role in the activation of proto-oncogenes and inactivation of tumor suppressor genes [32,34]. In addition, karyotypic alterations have been found in almost all types of neoplastic cells, and used as diagnostic indicators [35-37]. Apart from the carcinogenesis process being selective for such events, these aberrations are a hallmark of exposure of carcinogens that tend to be clastogenic. Hence, chromosomal aberrations can be effectively used as predictors of cancer or biomarkers of early detection.

\section{Multistep carcinogenesis: An opportunity for cancer prevention}

During the last 30 years, molecular biology has contributed greatly to our understanding of colorectal carcinogenesis. It is estimated that during this time period (decades to life long [38]) CRC carcinogenesis some 4 to 7 rate-limiting genetic changes must take place before a normal cell becomes malignant $[39,40]$. This chronic multistep evolutionary nature of CRC provides ample opportunity for early detection and prevention of cancer.

Genetic instability is the hallmark of molecular colorectal carcinogenesis. The genomic neoplastic continuum can be categorized as early, intermediate, and late events; which seems to proceed through at least two distinct pathways involving chromosomal instability (CIN) or microsatellite instability (MSI or MIN) [41]. Multiple genes are involved in the final histopathologic morphologic expression of CIN and/or MSI colorectal carcinomas (Fig. 1). Individual genetic variations (or polymorphisms) may result in the phenotypic differences, which are manifested by individuals and populations. The recognition, examination, and comparison of the CIN and MSI pathways provide insights into CRC pathogenesis.

\section{Early detection lessons learned from inherited cancers}

Our knowledge of molecular biomarkers for early cancer detection has greatly improved with understanding in the hereditary CRC. The majority of CRC are sporadic (or non-familial), occurring in individuals without familial adenomatous polyposis (FAP), hereditary nonpolyposis colorectal cancer (HNPCC), family 
history of CRC, or inflammatory bowel disease [42]. The molecular aberrations unveiled during and after the discovery of each hereditary gene discovery has advanced our knowledge of biomarkers as discovery of mutations in these and related genes grew several fold.

Familial adenomatous polyposis (FAP) and hereditary non-polyposis colorectal carcinoma (HNPCC) constitute "high-risk" prototypical models for majority of CRC cancers (sporadic and genetic) [43]. Similarly, each of these distinct pathways represent two major pathways of genetic instability observed in majority of colorectal carcinomas. In $80 \%$ to $85 \%$ of $\mathrm{CRC}$, the predominant carcinogenic pathway is demonstrated by aneuploidy, chromosomal rearrangements, and translocations characterized by chromosomal instability (CIN) [43,44]. The remaining $15 \%$ to $20 \%$ of CRC involve a second pathway of genetic instability that occurs at the nucleotide level, resulting from increased mutations at repetitive DNA sequence elements called microsatellites characterized by microsatellite instability (MSI or MIN). Each hereditary CRC syndrome are represented by a small percentage of overall CRC but contributed to the understanding of carcinogenesis tremendously.

FAP and the majority of sporadic CIN tumors arise from adenomatous polyps in the distal colon. In contrast, HNPCC and most sporadic tumors MIN develop in the proximal colon [43]. The precursor lesions of HNPCC and the sporadic MIN pathway are widely debated, recent evidence suggests that tumors with MSI may arise from non-adenomatous polyps $[45,46]$. Our understanding of HNPCC and its underlying genotype and phenotype has led to the use of MSI markers as the risk and early detection markers either independently or in conjunction with other markers (as a panel of markers) [29].

\section{Famialial adenomatous polyposis colorectal carcinoma}

FAP results from an autosomal dominant germline mutation in the adenomatous polyposis coli $(A P C)$ tumor suppressor gene $[47,48]$. Moreover, studies suggest that the same $A P C$ gene is somatically mutated in $70 \%$ of all sporadic CRC [32]. Other mutations that are subsequently accumulated in FAP and sporadic colorectal carcinomas include $k$-ras, $\beta$-catenin, SMAD 2/4, and p53. In FAP and sporadic CIN colorectal carcinomas, allelic loss or loss of heterozygosity ( $\mathrm{LOH}$ ) of the $A P C$ tumor suppressor gene is consistent with Knudson's "two-hit" hypothesis [49-51]. One germline allelic mutation is inherited in FAP while the second mutation is somatically-derived. On the other hand, two somatic mutations are required for colorectal carcinogenesis in sporadic CIN cancers. Hundreds to thousands of adenomatous polyps are seen at early age with additional gastrointestinal symptoms appearing in the third and fourth decades of life. Without removal of the colon, almost all FAP individuals develop CRC by 40 years of age.

It is believed that mutations in APC and k-ras initiate the growth of an adenoma. Transitions from the adenoma to carcinoma are correlated with subsequent alterations in $\beta$-catenin, SMAD 2/4, and p53. Invasive CRC develops when a sufficient number of mutated genes accumulate in the absence of their normal alleles [32].

\section{HNPCC and sporadic colorectal carcinoma with MSI}

HNPCC or Lynch syndrome is characterized by autosomal dominant germline mutations in the mismatch repair genes, i.e., $h M S H 2, h M S H 6, h M L H 1, h P M S 1$ or $h P M S 2$. HNPCC accounts for one-third or less of all MSI (or MIN) tumors [17], whereas the remaining two-thirds are due to sporadic somatic silencing of the $h M L H 1$ gene [52]. For a complete review of DNA mismatch repair see references $[14,53]$.

Pathologically, HNPCC lacks specific features, making it difficult to distinguish from sporadic CRC. Diagnosis is generally based upon a combination of clinical features and the Amsterdam or Bethesda criteria [48, 54]. HNPCC is defined by the presence of three or more family members with $\mathrm{CRC}$ in at least two successive generations, with at least one affected subject being diagnosed at less than 50 years of age. Average age of diagnosis is usually 41 years [55]. HNPCC is generally located in the proximal colon and is usually poorly differentiated with mucinous histopathology [56]. Germline mutations in $h M S H 2$ or $h M L H 1$ are found in $45 \%$ to $70 \%$ of all families that meet the strict criteria for HNPCC [57-59]. Current guideline for HNPCC, although is still under development has included histopathological and genetic markers into account for early detection and risk assessment.

Microsatellite instability is the hallmark of HNPCC and MSI pathway of colorectal carcinomas. Initially, three independent groups identified genomic instability leading to MSI carcinoma in $12-15 \%$ of sporadic 
CRCs [60-62]. Subsequent reports confirmed the presence of similar pathways in extra-colorectal carcinomas such as endometrial, ovarian, urinary tract, and upper gastrointestinal tract [63-65]. With refined molecular biology and biomarker research it is becoming clear that at least two different forms of MSI exists in CRC and different risks might be associated with each.

\section{Shifting paradigms in biomarkers research}

With recent improvement in the technologies that help to identify molecular and epidemiological knowledge of colorectal carcinogenesis and risk of developing CRC, effective chemoprevention of this common disease is a realistic proposition. Multiplexing of molecular analysis is providing detailed genetic, epigenetic and proteomic profiles of early lesions of colorectal cancer with increasing accuracy. If the current trend is an indication for future directions in the biomarker research we are in for an enthralling voyage. There seems to be a shift in the research paradigms in biomarker research, as the days of one sample one test seems to be numbered. Multiplexing and intimate dependence on the computers and use of algorithms to reliably predict the presence, risk or modulation in the state of preneoplastic and neoplastic disease.

\section{Proteomic expression profiling}

New technological advances are so stark in their impact that it is certain that their applications for cancer detection will have an important effect on public health; however, to achieve this goal, more specific and sensitive markers are needed. Detection of serum proteins via matrix-assisted laser desorption and ionization time-of-flight (MALDI-TOF) and surfaceenhanced laser desorption and ionization time-of-flight (SELDI-TOF) mass spectroscopy represent a profiling approach for early detection [66]. Serum protein profiles can provide thousands of data points, which can be further analyzed by sophisticated algorithms. Recently, a successful demonstration of this approach was published where using serum proteomic patterns successfully predicted ovarian cancer with $100 \%$ sensitivity, 95\% specificity and overall $94 \%$ predictive value. These findings lay a foundation for a new paradigm for the biomarkers of early detection and risk by justifying a prospective population based assessment of proteomic pattern technology as a screening tool for not only ovarian cancer but also for other cancers.

\section{Future directions}

Availability of new technologies is the single most important factor in the future of biomarkers for early detection of cancer. With the completion of human genome sequencing and a plethora of expression profiles, new information coming from the technologyderived methods can be of paramount importance in human colorectal cancer studies. Cancer biomarkers in near future has already begin to realize the role of susceptibility genes by more efficiently investigating their influence on specific effects of exposures and response to genotoxic agents are being elucidated. These considerations are even more valid when the contribution of proteomics is considered as well. The contribution of high throughput technologies to cancer biology is under intense investigation. However, there are serious challenges before new technologies can have a real impact as no evidence has been produced about the association between data on gene expression or proteins and classical markers of exposure or early effect. On the other hand this is the first step of a validation process as the population biomarker validation is not started yet.

Patterns of proteins from tissue, cell or serum defines the proteomic profile for an individual at a given time. Hence, proteomics can be used successfully to detect cancer at early stage of onset and even predict future propensity of an individual at risk. High-throughput proteomic approaches are already yielding a large number of new molecular biomarkers. For example, highthroughput mass spectroscopic techniques can now be used to compare profiles between normal, precancerous and cancerous lesions.

The future of cancer biomarkers is promising, as sophisticated analytical techniques are being developed to determine smaller and smaller amount of molecular determinants of the disease. By building reliable biomarker databases, a promising future is the integration of information from the genome programs to expand the scientific frontiers on causes, risk prediction and prevention of the disease.

\section{Conclusion}

Colorectal carcinogenesis is a continuum of molecular Darwinian selection events over several years to a decade long period. With this chronic molecular aberration come distinct phenotypic and genotypic stages that can be recognized clinically. Accumulation of alterations in the gene sequences, expression levels and 
protein structure and function have been associated with each stage (i.e, aberrant crypts, dysplasia, hyperplasia, adenoma and carcinoma) of colorectal carcinogenesis. For early detection and biomarker discovery each of these stages represents opportunity. By devising strategies for early detection and eliminating either by reducing risk factors or by monitoring these biomarkers after nutriceutical/pharmaceutical intervention. The utmost challenges are in the future as the knowledge of early biomarkers of CRC are validated and applied routinely in a clinical setting.

\section{References}

[1] H.T. Lynch, R.J. Thomas, H.A. Gurgis and J. Lynch, Clues to cancer risk: biologic markers, Am Fam Physician 11 (1975), 153-158.

[2] N.L. Petrakis and M.C. King, Genetic markers and cancer epidemiology, Cancer 39 (1977), 1861-1866.

[3] J.T. Galambos, Carcinoembryonic antigen and neoplastic disease, South Med J 66 (1973), 1216-1217.

[4] S. Hanash, F. Brichory and D. Beer, A proteomic approach to the identification of lung cancer markers, Dis Markers $\mathbf{1 7}$ (2001), 295-300.

[5] M.B. Jones, H. Krutzsch, H. Shu, Y. Zhao, L.A. Liotta, E.C. Kohn and E.F. Petricoin EF, 3rd., Proteomic analysis and identification of new biomarkers and therapeutic targets for invasive ovarian cancer, Proteomics 2 (2002), 76-84.

[6] T.J. Giordano, K.A. Shedden, D.R. Schwartz, R. Kuick, J.M Taylor, N. Lee, D.E. Misek, J.K. Greenson, S.L. Kardia, D.G. Beer, G. Rennert, K.R. Cho, S.B. Gruber, E.R. Fearon and $\mathrm{S}$. Hanash, Organ-specific molecular classification of primary lung, colon, and ovarian adenocarcinomas using gene expression profiles, Am J Pathol 159 (2001), 1231-1238.

[7] A.G.J. Knudson, Genetic predisposition to cancer, in: Origins of Human Cancer, (Book A), H.H. Hiat and J.A. Watson, eds, Cold Spring Harbor, New York, 1977, pp. 45-52.

[8] R. Doll and R. Peto, The causes of cancer: quantitative estimate of avoidable risks of cancer in the United States today, $J$ Natl Cancer Inst (1981), 66.

[9] M.B. Sporn, Chemoprevention of cancer, Lancet 342 (1993), 1211-1213.

[10] S. De Flora, C. Bennicelli and M. Bagnasco, Rationale and mechanisms of cancer chemoprevention, Recent Results Cancer Res 151 (1999), 29-44.

[11] P.R. Srinivas, B.S. Kramer and S. Srivastava, Trends in biomarker research for cancer detection, Lancet Oncol 2 (2001), 698-704

[12] S. Srivastava, M. Verma and D.E. Henson, Biomarkers for early detection of colon cancer, Clin Cancer Res 7 (2001), 1118-1126.

[13] J.D. Groopman and T.W. Kensler, The light at the end of the tunnel for chemical-specific biomarkers: daylight or headlight? Carcinogenesis 20 (1999), 1-11.

[14] A. Umar and T.A. Kunkel, DNA-replication fidelity, mismatch repair and genome instability in cancer cells, Eur J Biochem 238 (1996), 297-307.

[15] L.A. Loeb, C.F. Springgate and N. Battula, Errors in DNA replication as a basis of malignant changes, Cancer Res $\mathbf{3 4}$ (1974), 2311-2321.
[16] L.A. Loeb, A Mutator Phenotype in Cancer, Cancer Res 61 (2001), 3230-3239.

17] F.S. Leach, N.C. Nicolaides, N. Papadopoulos, B. Liu, J. Jen, R. Parsons, P. Peltomaki, P. Sistonen, L.A. Aaltonen and M. Nystrom-Lahti et al., Mutations of a mutS homolog in hereditary nonpolyposis colorectal cancer, Cell 75 (1993), 12151225.

[18] R. Fishel, M.K. Lescoe, M.R. Rao, N.G. Copeland, N.A. Jenkins, J. Garber, M. Kane and R. Kolodner, The human mutator gene homolog $\mathrm{MSH} 2$ and its association with hereditary nonpolyposis colon cancer, Cell 75 (1993), 1027-1038.

[19] A. Umar, J.C. Boyer, D.C. Thomas, D.C. Nguyen, J.I. Risinger, J. Boyd, Y. Ionov, M. Perucho and T.A. Kunkel, Defective mismatch repair in extracts of colorectal and endometrial cancer cell lines exhibiting microsatellite instability, $J$ Biol Chem 269 (1994), 14367-14370.

[20] R. Kaaks, Endogenous hormone metabolism as an exposure marker in breast cancer chemoprevention studies, IARC Sci Publ 154 (2001), 149-162.

[21] D.E. Henson, S. Srivastava and B.S. Kramer, Molecular and genetic targets in early detection, Curr Opin Oncol 11 (1999), 419-425.

[22] I. Petricoin, F. Emanuel, A.M. Ardekani, B.A. Hitt, P.J. Levine, V.A. Fusaro, S.M. Steinberg, G.B. Mills, C. Simone and D.A. Fishman, Use of proteomic patterns in serum to identify ovarian cancer, The Lancet 359 (2002), 572-577.

[23] P.E. Jackson and J.D. Groopman, Aflatoxin and liver cancer, Baillieres Best Pract Res Clin Gastroenterol 13 (1999), 545555.

[24] C.P. Wild and P.C. Turner, Exposure biomarkers in chemoprevention studies of liver cancer, IARC Sci Publ 154 (2001), 215-222.

[25] G.J. Kelloff, R. Lieberman, V.E. Steele, C.W. Boone, R.A. Lubet, L. Kopelovich, W.A. Malone, J.A. Crowell, H.R. Higley and C.C. Sigman, Agents, biomarkers, and cohorts for chemopreventive agent development in prostate cancer, Urology $\mathbf{5 7}$ (2001), 46-51.

[26] W.K. Hong and M.B. Sporn, Recent advances in chemoprevention of cancer, Science 278 (1997), 1073-1077.

[27] C.W. Boone and G.J. Kelloff, Biomarker end-points in cancer chemoprevention trials, IARC Sci Publ 142 (1997), 273-280.

[28] M.S. Tockman, Lung cancer: chemoprevention and intermediate effect markers, IARC Sci Publ 154 (2001), 257-270.

[29] D.A. Ahlquist, J.E. Skoletsky, K.A. Boynton, J.J. Harrington, D.W. Mahoney, W.E. Pierceall, S.N. Thibodeau and A.P. Shuber, Colorectal cancer screening by detection of altered human DNA in stool: feasibility of a multitarget assay panel, Gastroenterology 119 (2000), 1219-1227.

[30] D.A. Ahlquist and A.P. Shuber, Stool screening for colorectal cancer: evolution from occult blood to molecular markers, Clin Chim Acta 315 (2002), 157-168.

[31] E.R. Fearon and B. Vogelstein, A genetic model for colorectal tumorigenesis, Cell 61 (1990), 759-767.

[32] B. Vogelstein, E.R. Fearon, S.R. Hamilton, S.E. Kern, A.C. Preisinger, M. Leppert, Y. Nakamura, R. White, A.M. Smits and J.L. Bos, Genetic alterations during colorectal tumor development, N Engl J Med 319 (1988), 525-532.

[33] M. Ilyas, J. Straub, I.P. Tomlinson and W.F. Bodmer, Genetic pathways in colorectal and other cancers, Eur J Cancer 35 (1999), 1986-2002.

[34] J.C. Alers, P.J. Krijtenburg, A.N. Vis, R.F. Hoedemaeker, M.F. Wildhagen, W.C. Hop, T.T. van Der Kwast, F.H. Schroder, H.J. Tanke and H. van Dekken, Molecular cytogenetic analysis of prostatic adenocarcinomas from screening studies: early 
cancers may contain aggressive genetic features, Am J Pathol 158 (2001), 399-406.

[35] R.E. Crowell and S.A. Belinsky, Genetic changes in lung cancer: potential biomarkers for early detection and prevention, J Lab Clin Med 130 (1997), 550-557.

[36] M. Follen and D. Schottenfeld, Surrogate endpoint biomarkers and their modulation in cervical chemoprevention trials, Cancer 91 (2001), 1758-1776.

[37] H.T. Lynch, G.S. Schuelke and J.F. Lynch, Biomarker studies in hereditary ovarian carcinoma, Cancer Detect Prev 8 (1985), 129-134.

[38] D.W. Day, The adenoma-carcinoma sequence, Scand J Gastroenterol Suppl 104 (1984), 99-107.

[39] J.S. Bertram, The molecular biology of cancer, Mol Aspects Med 21 (2000), 167-223.

[40] M.J. Renan, How many mutations are required for tumorigenesis? Implications from human cancer data, Mol Carcinog 7 (1993), 139-146.

[41] C. Lengauer, K.W. Kinzler and B. Vogelstein, Genetic instabilities in human cancers, Nature 396 (1998), 643-649.

[42] S.J. Winawer, A quarter century of colorectal cancer screening: progress and prospects, J Clin Oncol 19 (2001), 6S-12S.

[43] A. Lindblom, Different mechanisms in the tumorigenesis of proximal and distal colon cancers, Curr Opin Oncol 13 (2001), 63-69.

[44] B. Liu, N.C. Nicolaides, S. Markowitz, J.K. Willson, R.E. Parsons, J. Jen, N. Papadopolous, P. Peltomaki, A. de la Chapelle and S.R. Hamilton et al., Mismatch repair gene defects in sporadic colorectal cancers with microsatellite instability, Nat Genet 9 (1995), 48-55.

[45] M. Stolte and B. Bethke, Colorectal mini-de novo carcinoma: a reality in Germany too, Endoscopy 27 (1995), 286-290.

[46] N.J. Hawkins and R.L. Ward, Sporadic colorectal cancers with microsatellite instability and their possible origin in hyperplastic polyps and serrated adenomas, J Natl Cancer Inst $\mathbf{9 3}$ (2001), 1307-1313.

[47] K.W. Kinzler, M.C. Nilbert, L.K. Su, B. Vogelstein, T.M. Bryan, D.B. Levy, K.J. Smith, A.C. Preisinger, P. Hedge and D. McKechnie et al., Identification of FAP locus genes from chromosome 5q21, Science 253 (1991), 661-665.

[48] C.R. Boland, S.N. Thibodeau, S.R. Hamilton, D. Sidransky, J.R. Eshleman, R.W. Burt, S.J. Meltzer, M.A. RodriguezBigas, R. Fodde, G.N. Ranzani and S. Srivastava, A National Cancer Institute Workshop on Microsatellite Instability for cancer detection and familial predisposition: development of international criteria for the determination of microsatellite instability in colorectal cancer, Cancer Res 58 (1998), 52485257.

[49] A.G. Knudson, Jr., Mutation and cancer: statistical study of retinoblastoma, Proc Natl Acad Sci USA 68 (1971), 820-823.

[50] E.R. Fearon and B. Vogelstein, A genetic model for colorectal tumorigenesis, Cell 61 (1990), 759-767.

[51] W.K. Cavenee, Loss of heterozygosity in stages of malignancy, Clin Chem 35 (1989), B48-B52.

[52] J.G. Herman, A. Umar, K. Polyak, J.R. Graff, N. Ahuja, J.P. Issa, S. Markowitz, J.K. Willson, S.R. Hamilton, K.W. Kinzler, M.F. Kane, R.D. Kolodner, B. Vogelstein, T.A. Kunkel and S.B. Baylin, Incidence and functional consequences of
hMLH1 promoter hypermethylation in colorectal carcinoma, Proc Natl Acad Sci USA 95 (1998), 6870-6875.

[53] G. Marra and C.R. Boland, DNA repair and colorectal cancer, Gastroenterol Clin North Am 25 (1996), 755-772.

[54] M.A. Rodriguez-Bigas, C.R. Boland, S.R. Hamilton, D.E. Henson, J.R. Jass, P.M. Khan, H. Lynch, M. Perucho, T. Smyrk, L. Sobin and S. Srivastava, A National Cancer Institute Workshop on Hereditary Nonpolyposis Colorectal Cancer Syndrome: meeting highlights and Bethesda guidelines, $J$ Natl Cancer Inst 89 (1997), 1758-1762.

[55] A. Muller and R. Fishel, Mismatch repair and the hereditary non-polyposis colorectal cancer syndrome (HNPCC), Cancer Invest 20 (2002), 102-109.

[56] S.R. Hamilton, Origin of colorectal cancers in hyperplastic polyps and serrated adenomas: another truism bites the dust, J Natl Cancer Inst 93 (2001), 1282-1283.

[57] G. Marra and C.R. Boland, Hereditary nonpolyposis colorectal cancer: the syndrome, the genes, and historical perspectives, J Natl Cancer Inst 87 (1995), 1114-1125.

[58] J.T. Wijnen, H.F. Vasen, P.M. Khan, A.H. Zwinderman, H. van der Klift, A. Mulder, C. Tops, P. Moller and R. Fodde, Clinical findings with implications for genetic testing in families with clustering of colorectal cancer, N Engl J Med 339 (1998), 511-518.

[59] B. Liu, R. Parsons, N. Papadopoulos, N.C. Nicolaides, H.T. Lynch, P. Watson, J.R. Jass, M. Dunlop, A. Wyllie, P. Peltomaki, A. de la Chapelle, S.R. Hamilton, B. Vogelstein and K.W. Kinzler, Analysis of mismatch repair genes in hereditary non-polyposis colorectal cancer patients, Nat Med 2 (1996), 169-174.

[60] L.A. Aaltonen, P. Peltomaki, F.S. Leach, P. Sistonen, L. Pylkkanen, J.P. Mecklin, H. Jarvinen, S.M. Powell, J. Jen and S.R. Hamilton et al., Clues to the pathogenesis of familial colorectal cancer, Science 260 (1993), 812-816.

[61] P. Peltomaki, R.A. Lothe, L.A. Aaltonen, L. Pylkkanen, M. Nystrom-Lahti, R. Seruca, L. David, R. Holm, D. Ryberg and A. Haugen et al., Microsatellite instability is associated with tumors that characterize the hereditary non-polyposis colorectal carcinoma syndrome, Cancer Res 53 (1993), 5853-5855.

[62] Y. Ionov, M.A. Peinado, S. Malkhosyan, D. Shibata and M. Perucho, Ubiquitous somatic mutations in simple repeated sequences reveal a new mechanism for colonic carcinogenesis, Nature 363 (1993), 558-561.

[63] H.T. Lynch and J.F. Lynch, Familial factors and genetic predisposition to cancer: population studies, Cancer Detect Prev 15 (1991), 49-57.

[64] H.T. Lynch, T. Smyrk and J.F. Lynch, Molecular genetics and clinical-pathology features of hereditary nonpolyposis colorectal carcinoma (Lynch syndrome): historical journey from pedigree anecdote to molecular genetic confirmation, Oncology 55 (1998), 103-108.

[65] H.T. Lynch, T. Smyrk and J.R. Jass, Hereditary nonpolyposis colorectal cancer and colonic adenomas: aggressive adenomas? Semin Surg Oncol 11 (1995), 406-410.

[66] P.R. Srinivas, S. Srivastava, S. Hanash and G.L. Wright, Jr., Proteomics in early detection of cancer, Clin Chem 47 (2001), 1901-1911. 


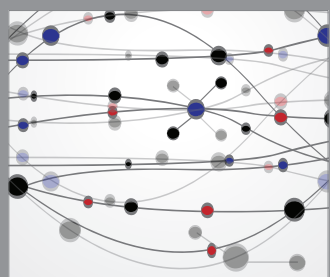

The Scientific World Journal
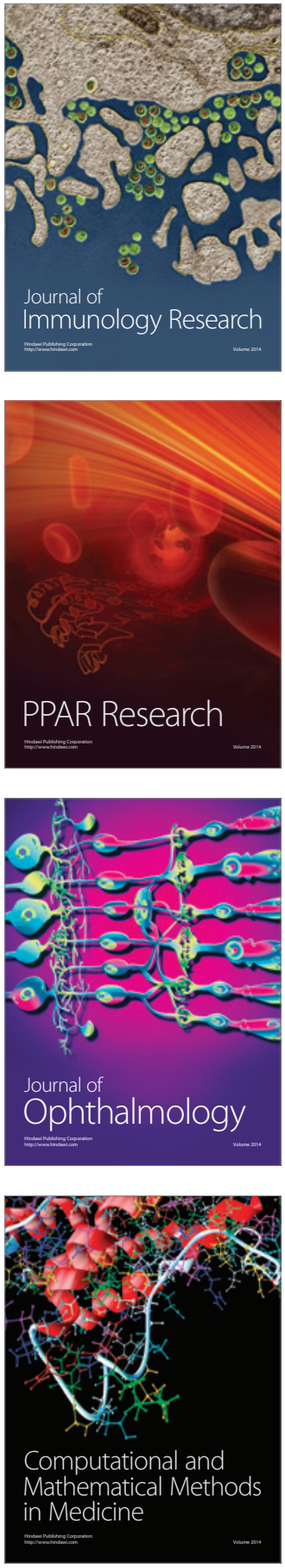

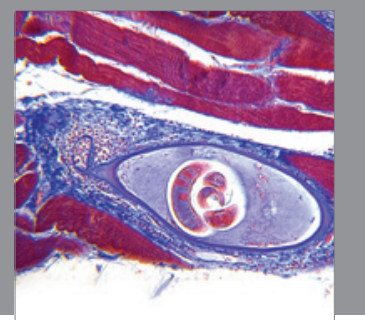

Gastroenterology

Research and Practice
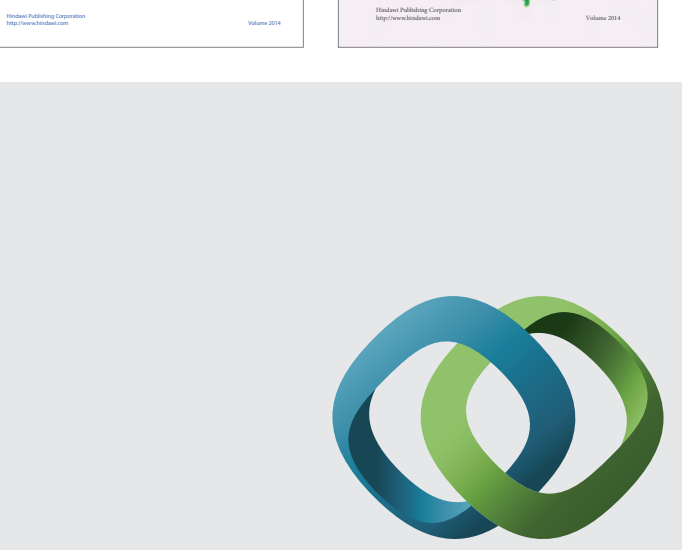

\section{Hindawi}

Submit your manuscripts at

http://www.hindawi.com
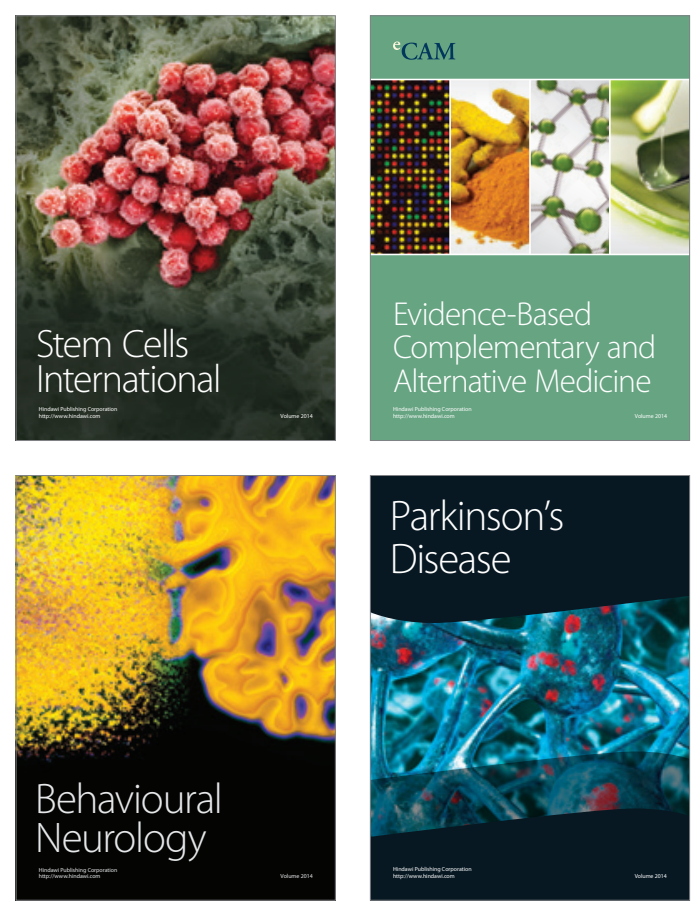

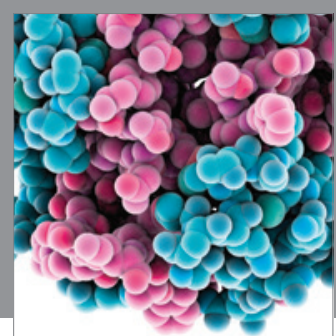

Journal of
Diabetes Research

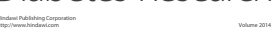

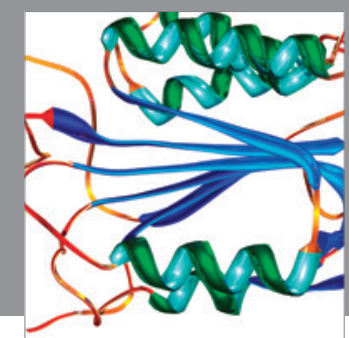

Disease Markers
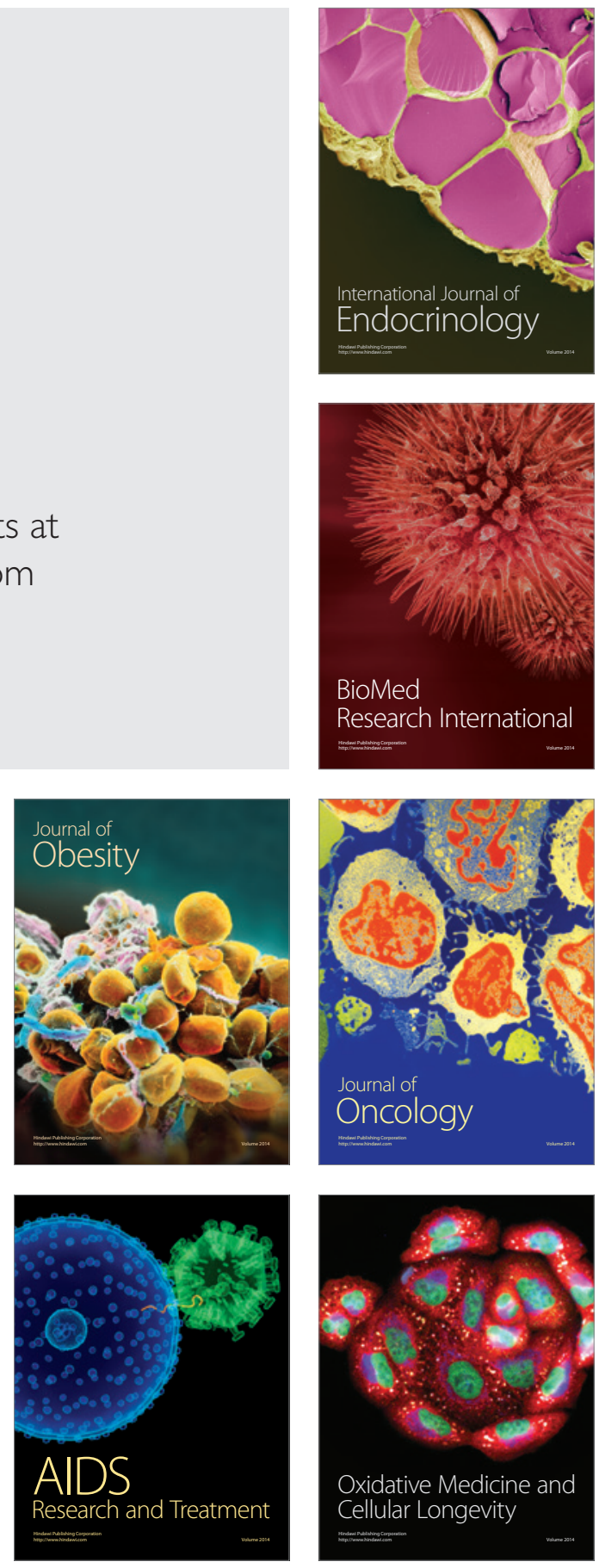